\title{
ENFERMEDAD VERTEBRAL DEGENERATIVA EN LA COLECCIÓN DEL CEMENTERIO CENTRAL DE BOGOTÁ, COLOMBIA (SIGLOS XIX Y XX)
}

\author{
VERTEBRAL DEGENERATIVE DISEASE IN THE COLLECTION OF THE \\ CENTRAL CEMETERY OF BOGOTÁ, COLOMBIA (19TH AND 2OTH CENTURIES)
}

\author{
Fernando Suescún ${ }^{1}$ y Claudia Mercedes Rojas-Sepúlveda ${ }^{2 *}$
}

\begin{abstract}
El patrón de Enfermedad Vertebral Degenerativa (EVD) fue registrado en 192 esqueletos del Globo B del Cementerio Central de Bogotá, datados entre mediados del siglo XIX y principios del siglo XX. El contexto arqueológico y la documentación histórica sugieren que personas con bajo estatus socioeconómico fueron enterradas en esta área. En total, 3.258 vértebras fueron examinadas. Para los cuerpos vertebrales se registró osteofitos, cambio del contorno articular (labiación) y porosidad. Para las facetas articulares se observó cambio del contorno articular, porosidad y eburnación. Se diagnosticó la enfermedad siempre que estuvieran presentes las lesiones, excluyendo la porosidad. Se usaron dos métodos de cálculo de frecuencia, uno, por individuo y otro, por vértebra. El $76 \%$ de los individuos estudiados fueron clasificados como positivos. La alta prevalencia en individuos jóvenes $(39,39 \%)$ sugiere que esta población tuvo un estilo de vida extenuante desde temprana edad. Diferencias en el patrón de las lesiones fueron halladas entre los sexos, particularmente en el número de vértebras afectadas, y pueden estar relacionadas con diferentes tipos de actividades reportadas en los documentos históricos.
\end{abstract}

Palabras claves: bioarqueología, actividad física, estilo de vida, población histórica, paleoepidemiología, enfermedad vertebral degenerativa.

The pattern of vertebral degenerative disease (VDD) was recorded in 192 skeletons from mid-19th to early 20th century at the Central Cemetery of Bogotá, Globe B. Archaeological context and historical documentation suggest that people with low socio-economic status were buried in this location. In total, 3,258 vertebrae were examined; osteophytes, joint surface contour change (lipping), and pitting were recorded in vertebral bodies, while joint surface contour change, pitting, and eburnation were observed in apophyseal joints. The disease was regarded present when any of the lesions, excluding pitting, was observed. Two methods of frequency calculation were used: one by individual and the other by vertebra; seventy-six percent of the individuals were classified as positive. High prevalence in young individuals (39.39\%) suggests that this population had a strenuous lifestyle since childhood. Pattern differences of lesions found between the sexes, namely the number of affected vertebrae, may be related to different types of activities reported in historical documentation.

Key words: Bioarchaeology, physical activity, lifestyle, historical population, paleoepidemiology, vertebral degenerative disease.

La Enfermedad Articular Degenerativa (EAD) es la degeneración heterogénea y dinámica de toda la articulación como resultado de un desequilibrio entre la ruptura y la reparación de los tejidos articulares (Hunter y Felson 2006; Litwic et al. 2013). Es la enfermedad de las articulaciones más prevalente a nivel mundial (Song et al. 2006) y la más observada en colecciones arqueológicas (Bridges 1992; Rogers y Waldron 1995; Ortner 2003). LaEAD es multifactorial y sus agentes de riesgo se clasifican en factores sistémicos tales como la edad, el sexo y la genética; y en factores biomecánicos locales como la obesidad, los traumas y el estrés biomecánico (Bridges 1992; Dieppe 1995; Jurmain 1977, 1980; Jurmain y Kilgore 1995; Ortner 2003; Rogers y Waldron 1995). La pertinencia de la EAD como indicador de actividad física es debatida. Algunos investigadores asocian el deterioro articular con el estrés biomecánico (Bridges 1991, 1992; Sofaer 2000; RojasSepúlveda et al. 2008), mientras otros argumentan que los factores determinantes son la edad (Rogers et al. 1987; Waldron 1991, 1994) y las constricciones biológicas (Knüsel et al. 1997).

\footnotetext{
${ }^{1}$ Fundación Erigaie, Bogotá, Colombia. Ifsuescuna@unal.edu.co

${ }^{2}$ Departamento de Antropología, Facultad de Ciencias Humanas, Universidad Nacional de Colombia, Bogotá, Colombia. *autora correspondiente: cmrojass@unal.edu.co
} 
Los cambios degenerativos en la columna vertebral -o Enfermedad Vertebral Degenerativa (EVD)- han sido ampliamente estudiados en bioarqueología, y varias investigaciones sugieren que pueden estar relacionados con el estilo de vida y el nivel de actividad física (Bridges 1994; Lovell 1994; Merbs 1983; Rojas-Sepúlveda et al. 2008; Stirland y Waldron 1997; Trinkaus 1985). Por su parte, algunos autores proponen que el bipedismo determina el patrón de distribución de la EVD, por lo que la columna vertebral no sería una estructura idónea para examinar cambios óseos relacionados con la actividad física (Knüsel et al. 1997). Sumado a esto, los estudios sobre la EAD suelen no ceñirse a un estándar de registro, ya que los métodos de diagnóstico y el tratamiento de los datos varían en cada investigación, lo cual frecuentemente dificulta comparar los resultados y replicar los métodos (Rojas-Sepúlveda et al. 2008).

A razón de que una actividad puede afectar de maneras diversas cada articulación (Jurmain 1991), no siempre es posible hacer una conexión directa entre EVD y labores específicas. Para hacer este tipo de inferencias sería necesario examinar todo el material óseo sin centrarse de manera exclusiva en una articulación (Jurmain 1980, 1991). Solo algunos estudios han logrado relacionar actividades a determinados patrones de EVD, gracias al cruce de datos osteológicos con la información arqueológica, etnográfica e histórica (Bridges 1994; Merbs 1983; Sofaer 2000). Con estas limitaciones en mente, el estudio de EVD en colecciones arqueológicas e históricas generalmente está orientado a ampliar la información disponible sobre la forma en que vivieron ciertos grupos humanos, así como a entender el impacto que dicho estilo de vida pudo tener sobre los individuos (Rojas-Sepúlveda 2009), más que a relacionar patrones de EVD con oficios específicos.

Con todo, el estudio paleoepidemiológico de la EVD ha permitido aproximarse a las condiciones y al estilo de vida de poblaciones de distintos períodos y áreas geográficas. Para el período histórico, se han analizado desde marinos ingleses del siglo XVI (Stirland y Waldron 1997) hasta esclavos afroamericanos del siglo XIX (Rathburn 1987), entre otras poblaciones (Knüsel et al. 1997; Lai y Lovell 1992; Novak y Šlaus 2011; Sofaer 2000; Waldron 1991; Watkins 2012; Woo y Pak 2014; Zampetti et al. 2016). En colecciones históricas es especialmente importante integrar los resultados osteológicos con la documentación histórica y arqueológica (Sofaer 2000).

En Latinoamérica la bioarqueología de la EAD aún es incipiente. Lleva poco más de 10 años y se ha centrado en poblaciones prehispánicas (Andrade et al. 2014; Llagostera 2017; López-Barrales et al. 2015; Mazza 2015; Mendonça y Arrieta 2011; Pechenkina y Delgado 2006; Ponce 2010; Quevedo 2000; RojasSepúlveda y Dutour 2014; Rojas-Sepúlveda et al.
2008; Scabuzzo 2012; Suby y Giberto 2019). Son pocas las investigaciones sobre esta enfermedad en contextos históricos (Giannotti 2016; Moreno Estefanell et al. 2018) y generalmente hacen parte de casos de estudio y revisiones paleopatológicas generales (Klaus 2016; Klaus et al. 2009; Mansegosa 2016; Martín et al. 2009; Rivera-Sandoval 2014; Silva-Pinto et al. 2017; Tiesler et al. 2010; Ubelaker 1994). La presente investigación pretende contribuir tanto al debate sobre la pertinencia de la EAD como indicador de actividad física, como al estudio de la EVD en contextos históricos de Latinoamérica.

\section{Materiales y Métodos}

\section{La muestra}

El Cementerio Central de Bogotá, Colombia (Figura 1), fue puesto en servicio en 1836 y funcionó hasta mediados del siglo XX. En el transcurso de su uso como espacio de sepultura fue dividido espacialmente en "Globo A", el camposanto de la población pudiente, y "Globo B", el cementerio de los pobres (Calvo 1998; Rojas-Sepúlveda y Rodríguez 2017). Parte del Globo B fue excavado entre 2009 y 2012 por el Equipo Colombiano de Investigaciones Antropológico-Forenses (ECIAF), cuyo análisis abordó el 19\% del material arqueológico; mientras que entre 2015 y 2016 un equipo de la Universidad Nacional de Colombia constituyó una colección con el $81 \%$ del material excavado, la cual alcanzó un número mínimo de individuos de 7.103, contando los de contextos mezclados. Gran parte de la colección corresponde a población mestiza (Rojas-Sepúlveda y Rodríguez 2017). A pesar de que la Ciudad cuenta con un registro (Libros Necrológicos) de los individuos sepultados en el Cementerio Central de Bogotá entre 1900 y 1938, los cuales incluyen información general de cada difunto (nombre, sexo y edad), su ocupación y domicilio (barrio de residencia), y causa de muerte, no es posible asociar los registros a los individuos o contextos que hacen parte de la colección. Actualmente se avanza en la consulta de estos archivos (Rojas-Sepúlveda y Suescún 2018).

Como criterio general de selección de la muestra, los individuos debían proceder de contextos no mezclados (Rojas-Sepúlveda 2009). El 24,58\% (1.746 individuos) de la colección cumplió con este criterio. Siguiendo las observaciones de Jurmain et al. (2012), se definieron los siguientes criterios específicos: sexo, edad, preservación ósea y condiciones patológicas previas. Primeramente, se eligieron individuos que contaran con más del $25 \%$ de la columna vertebral (seis vertebras). Seguidamente, se descartaron los individuos que presentaban traumas y condiciones patológicas 


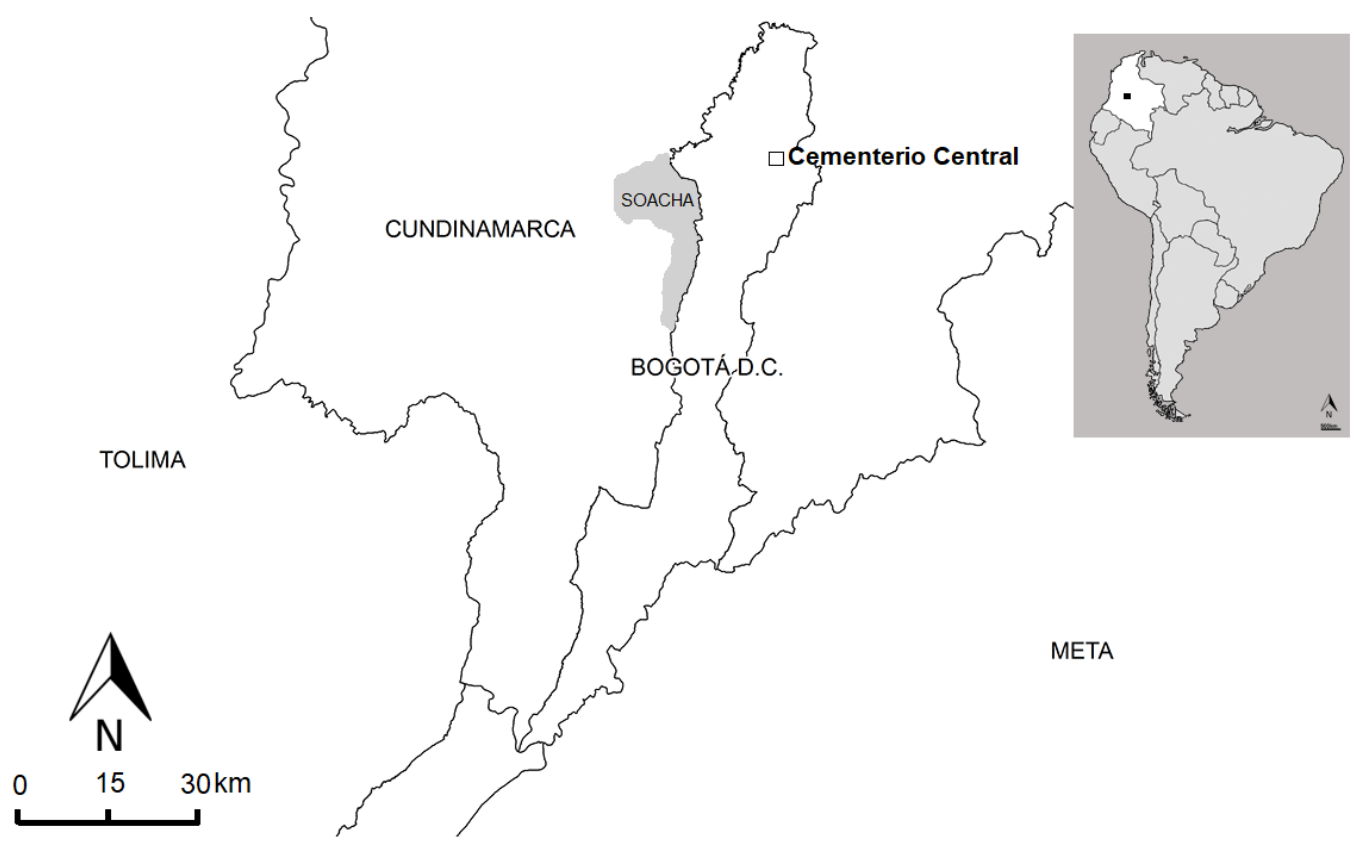

Figura 1. Mapa mostrando la ubicación del Cementerio Central de Bogotá, Colombia.

Map showing the location of the Central Cemetery of Bogotá, Colombia.

como hiperostosis esquelética idiopática difusa (DISH), espondilitis anquilosante (AS) o tuberculosis (TB), ya que tanto los traumatismos como determinadas patologías vertebrales pueden causar EAD o incrementar su grado de expresión en las articulaciones (Jurmain et al. 2012; Rojas-Sepúlveda 2009).

La literatura especializada propone que entre $15 \mathrm{y}$ 60 años es el rango de edad óptimo para observar las manifestaciones de EVD; mayores de 15 años, puesto que las estructuras para determinar el sexo del individuo están más desarrolladas; y menores de 60 años, ya que a partir de esta edad se incrementa el deterioro articular debido al proceso de envejecimiento (Cooper et al. 1994; Jurmain et al. 2012; Lovell 1994; RojasSepúlveda 2009). El sexo y la edad fueron observados morfológicamente (Bridges 1992, 1994), por lo que se emplearon métodos antropológicos macroscópicos (Brooks y Suchey 1990; Buikstra y Ubelaker 1994; Isçan et al. 1984, 1985; Meindl y Lovejoy 1985). En la Tabla 1 se observa la composición de la muestra por sexo y grupos etarios, según los rangos recomendados por Buikstra y Ubelaker (1994).

\section{Registro osteológico}

Se efectuó una sistemática evaluación de la preservación de las vértebras y de las manifestaciones de EVD, siguiendo el procedimiento propuesto por Rojas-
Tabla 1. Distribución por sexo y edad de la muestra. Age and sex composition of the sample.

\begin{tabular}{lcccc}
\hline & $\begin{array}{c}\text { Joven } \\
(15-30)\end{array}$ & $\begin{array}{c}\text { Medio } \\
(30-45)\end{array}$ & $\begin{array}{c}\text { Mayor } \\
(45+)\end{array}$ & Total \\
\hline Femenino & 20 & 74 & 23 & 117 \\
Masculino & 13 & 50 & 12 & 75 \\
Total & 33 & 124 & 35 & 192 \\
\hline
\end{tabular}

Sepúlveda et al. (2008). Las manifestaciones de EVD fueron observadas macroscópicamente. Debido a que la columna vertebral presenta dos tipos de articulaciones que difieren en su anatomía (Resnick 2002), las manifestaciones de EVD se registraron separadamente para cuerpos vertebrales y facetas articulares. En los cuerpos vertebrales se examinó osteofitos, cambio del contorno articular (labiación), y porosidad (Figura 2). En las facetas articulares se observó cambio del contorno articular (labiación), porosidad y eburnación (Figura 3).

La remodelación articular se refiere a neoformaciones óseas en los márgenes articulares, cuya estructura histológica es la de un tejido óseo más compacto que la del resto del hueso (Nathan 1962; Rogers y Waldron 1995). Para cuerpos vertebrales se hace distinción entre osteofitos y cambio del contorno articular; los osteofitos se manifiestan como proyecciones curvas focalizadas o "picos de loro", mientras que el cambio de contorno se 


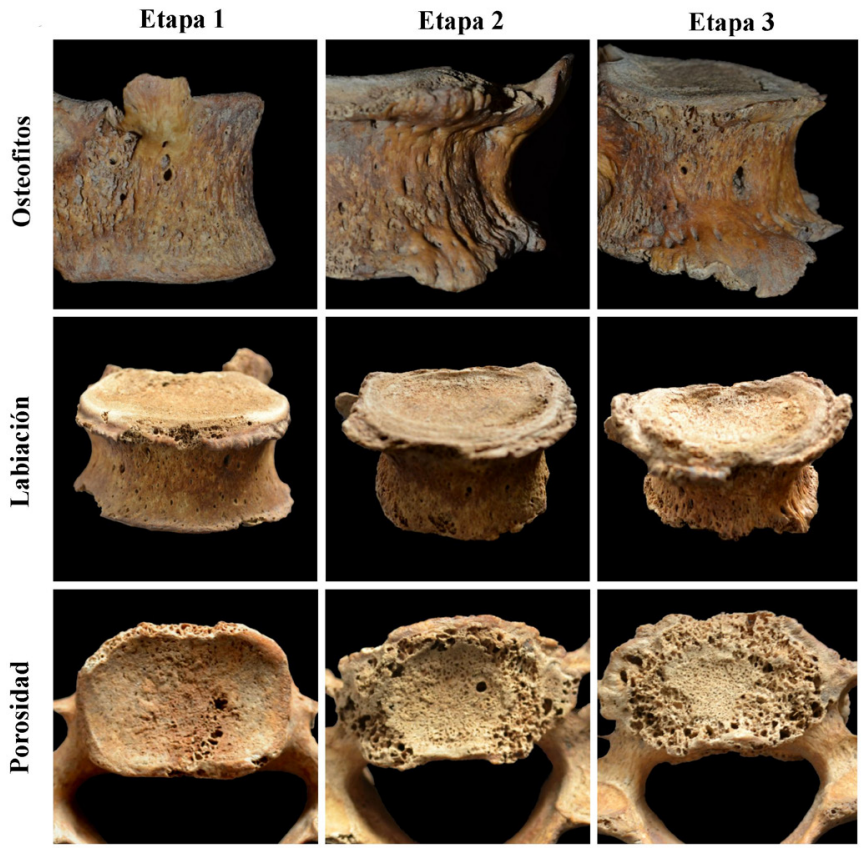

Figura 2. Manifestaciones de EVD (Enfermedad Vertebral Degenerativa) en las facetas articulares.

Manifestations of VDD (Vertebral Degenerative Disease) in the apophyseal joints.
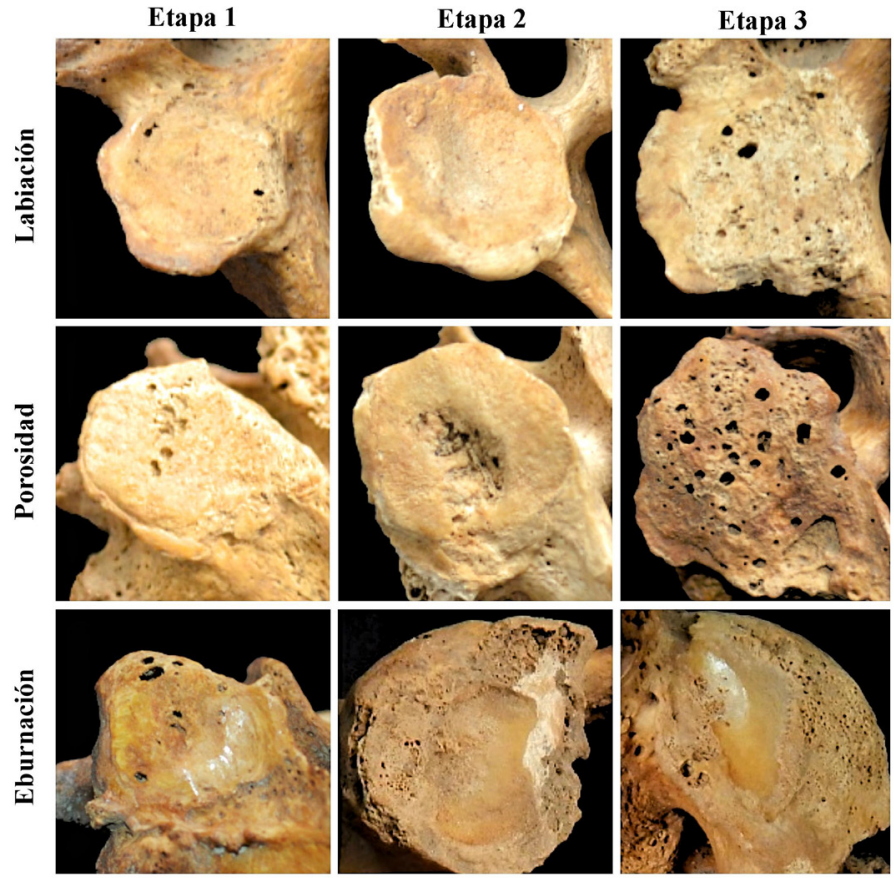

Figura 3. Manifestaciones de EVD (Enfermedad Vertebral Degenerativa) en el cuerpo vertebral.

Manifestations of VDD (Vertebral Degenerative Disease) in the vertebral body. 
caracteriza por el alargamiento de la superficie articular y la formación de un anillo en el borde de la articulación (Rogers et al. 1987). La porosidad corresponde a lesiones cavitadas en la superficie articular (Rothschild 1997). Estas lesiones, también referidas en la literatura clínica como quistes en el hueso subcondral, son focalizadas y poco profundas, y evolucionan en lesiones de mayor extensión y profundidad (Gellhorn et al. 2013; Li et al. 2013). La eburnación es la lesión patognomónica de la Enfermedad Articular Degenerativa (Rogers y Waldron 1995). Es producto de la fricción de las superficies óseas de una articulación, luego de la pérdida de la cápsula cartilaginosa que las recubre (Doherty et al. 2016). En el hueso seco se observa como una o más áreas pulidas y con brillo, presentando una apariencia similar al marfil (Doherty et al. 2016; Rogers y Waldron 1995; RojasSepúlveda 2009).

Las manifestaciones fueron evaluadas en cada superficie articular con una codificación de 0 a 3 . La codificación es una manera de reconocer el aspecto degenerativo de la enfermedad y su variabilidad (Nathan 1962; Rojas-Sepúlveda et al. 2008). El código 0 es para ausente; 1 para levemente visible; 2 para moderada; y 3 para severa. Para manifestaciones muy severas como anquilosamientos se emplea el código 4 y para pieza óseas deterioradas o ausentes "no observable" (Rojas-Sepúlveda et al. 2008). La Tabla 2 presenta las características osteológicas de la EVD con puntuaciones asociadas.

Tabla 2. Método de registro para codificar las manifestaciones de la EVD (Enfermedad Vertebral Degenerativa) (Suescún 2017:36).

Recording method for scoring VDD (Vertebral Degenerative Disease) features (Suescún 2017:36).

\begin{tabular}{|c|c|c|c|c|}
\hline Cód. & Porosidad & Osteofitos & Labiación & Eburnación \\
\hline 0 & Ausente & Ausente & Ausente & Ausente \\
\hline 1 & Reducida & $\begin{array}{c}\text { Puntos de } \\
\text { hiperostosis }\end{array}$ & $\begin{array}{l}\text { Ligera. Área de } \\
<1 / 3\end{array}$ & $\begin{array}{l}\text { Área menor } \\
\text { a } 1 / 3 \text {. }\end{array}$ \\
\hline 2 & Extensiva & $\begin{array}{l}\text { Protrusiones } \\
\text { horizontales }\end{array}$ & $\begin{array}{l}\text { Definida. Área } \\
\text { de } 1 / 3 \text { a } 2 / 3\end{array}$ & $\begin{array}{l}\text { Área de } \\
1 / 3 \text { a 2/3. } \\
\text { Surcos. }\end{array}$ \\
\hline 3 & Generalizada & $\begin{array}{l}\text { Proyecciones } \\
\text { curvas }\end{array}$ & $\begin{array}{l}\text { Considerable. } \\
\text { Área de }>2 / 3\end{array}$ & $\begin{array}{l}\text { Extensa } \\
\text { área pulida } \\
\text { similar } \\
\text { al marfil. } \\
\text { Estriaciones. }\end{array}$ \\
\hline 4 & & Anquilosamiento & Anquilosamiento & \\
\hline
\end{tabular}

El registro fue hecho por un observador (Suescún), a partir de un acompañamiento por parte de una de las autoras del método utilizado (Rojas-Sepúlveda) y del apoyo en escalas visuales (Figuras 2 y 3 ) y descripciones (Tabla 2), para evitar tanto el error interobservador como el error intra-observador (Waldron y Rogers 1991; Zampetti et al. 2016).

\section{Diagnóstico y análisis estadístico}

Para el diagnóstico de la EVD se decidió utilizar un método replicable aplicado previamente en colecciones óseas latinoamericanas, con el objetivo de facilitar la comparación de los resultados. La metodología aplicada fue "Porosidad excluida" (Rojas-Sepúlveda et al. 2008), la cual se basa en el planteamiento de que la porosidad no está directamente relacionada con la EAD (Rothschild 1997; Sofaer 2000). En consecuencia, si al menos una de las manifestaciones está presente en una articulación, excluyendo la porosidad, el diagnóstico de EVD es positivo. El método evita la sobreestimación al ignorar la porosidad como criterio de diagnóstico y evita la subestimación pues no requiere asociación entre manifestaciones (Rojas-Sepúlveda et al. 2008).

Se emplearon dos métodos para calcular la frecuencia de las manifestaciones y la prevalencia de la EVD: la Frecuencia por individuo (FI) y la Frecuencia por vértebra $(\mathrm{FV})$. FI es la proporción de columnas vertebrales afectadas, mientras que $\mathrm{FV}$ es la proporción de articulaciones y vértebras afectadas (Rojas-Sepúlveda et al. 2008).

Con el fin de evaluar las diferencias entre grupos por sexo y edad, se realizaron pruebas de Chi cuadrado $\left(\chi^{2}\right)$; y de Fisher, para valores esperados menores a cinco. Estas pruebas son utilizadas con frecuencia en estudios sobre EVD y otras condiciones patológicas, ya que permiten observar diferencias en la prevalencia de EVD entre grupos de sexo y edad, así como ha sido utilizado el p-value de 0,05 para el corte de significancia (RojasSepúlveda et al. 2008; Sofaer 2000; Zampetti et al. 2016).

\section{Resultados}

\section{Preservación}

En general, la vértebra más registrada es L1 $(79,69 \%, 153 / 192)$ mientras que C5 $(57,81 \%, 111 / 192)$ es la menos observada (Tabla 3). La región lumbar es la mejor representada con 741 vértebras $(77,18 \%$, asumiendo cinco lumbares por individuo). Le sigue la región torácica con 1.502 vértebras $(65,19 \%$, asumiendo 12 torácicas por individuo) y la región cervical, con 875 vértebras $(65,10 \%$, asumiendo siete cervicales por individuo). La primera vértebra sacra fue hallada en 140 columnas vertebrales $(72,92 \%$, asumiendo una primera vértebra sacra por individuo). En total fueron analizadas 3.258 vértebras.

En los individuos masculinos la vértebra más registrada fue $\mathrm{L} 1(66 / 75,88,00 \%)$, mientras que en los individuos de sexo femenino la vértebra más frecuente fue L5 (92/117, 78,63\%). La Tabla 3 presenta los porcentajes de preservación vertebral por grupos de sexo y edad. 
Tabla 3. Preservación de la columna vertebral por grupos de edad y sexo. En negrita, la vértebra mejor representada por grupo.

Preservation of the vertebral column by age and sex groups. In bold, the vertebra best represented by group.

\begin{tabular}{|c|c|c|c|c|c|c|c|c|c|c|c|c|c|}
\hline & & \multicolumn{2}{|c|}{ General } & \multicolumn{2}{|c|}{ Femenino } & \multicolumn{2}{|c|}{ Masculino } & \multicolumn{2}{|c|}{ Joven $(15-30)$} & \multicolumn{2}{|c|}{ Medio (30 - 45) } & \multicolumn{2}{|c|}{ Mayor (45+) } \\
\hline & & $n$ & $\%$ & $n$ & $\%$ & $n$ & $\%$ & $n$ & & $n$ & $\%$ & $n$ & $\%$ \\
\hline \multirow{7}{*}{$\frac{\frac{\tilde{s}}{\pi}}{\sum_{0}^{\frac{0}{0}}}$} & $\mathrm{C} 1$ & $119 / 192$ & 61,98 & $73 / 117$ & 62,39 & $46 / 75$ & 61,33 & $22 / 33$ & 66,67 & $71 / 124$ & 57,26 & $26 / 35$ & 74,29 \\
\hline & $\mathrm{C} 2$ & $136 / 192$ & 70,83 & $85 / 117$ & 72,65 & $51 / 75$ & 68,00 & $24 / 33$ & 72,73 & $87 / 124$ & 70,16 & $25 / 35$ & 71,43 \\
\hline & $\mathrm{C} 3$ & $138 / 192$ & 71,88 & $86 / 117$ & 73,50 & $52 / 75$ & 69,33 & $23 / 33$ & 69,70 & $85 / 124$ & 68,55 & $30 / 35$ & 85,71 \\
\hline & $\mathrm{C} 4$ & $127 / 192$ & 66,15 & $76 / 117$ & 64,96 & $51 / 75$ & 68,00 & $23 / 33$ & 69,70 & $74 / 124$ & 59,68 & $30 / 35$ & 85,71 \\
\hline & $\mathrm{C} 5$ & $111 / 192$ & 57,81 & $67 / 117$ & 57,26 & $44 / 75$ & 58,67 & $18 / 33$ & 54,55 & $69 / 124$ & 55,65 & $24 / 35$ & 68,57 \\
\hline & C6 & $122 / 192$ & 63,54 & $69 / 117$ & 58,97 & $53 / 75$ & 70,67 & $22 / 33$ & 66,67 & $77 / 124$ & 62,10 & $23 / 35$ & 65,71 \\
\hline & $\mathrm{C} 7$ & $122 / 192$ & 63,54 & $68 / 117$ & 58,12 & $54 / 75$ & 72,00 & $21 / 33$ & 63,64 & $81 / 124$ & 65,32 & $20 / 35$ & 57,14 \\
\hline \multirow{12}{*}{ 莺 } & $\mathrm{T} 1$ & $110 / 192$ & 57,29 & $66 / 117$ & 56,41 & $44 / 75$ & 58,67 & $21 / 33$ & 63,64 & $66 / 124$ & 53,23 & $23 / 35$ & 65,71 \\
\hline & $\mathrm{T} 2$ & $145 / 192$ & 75,52 & $89 / 117$ & 76,07 & $56 / 75$ & 74,67 & $23 / 33$ & 69,70 & $91 / 124$ & 73,39 & $31 / 35$ & 88,57 \\
\hline & $\mathrm{T} 3$ & $141 / 192$ & 73,44 & $82 / 117$ & 70,09 & $59 / 75$ & 78,67 & $24 / 33$ & 72,73 & $87 / 124$ & 70,16 & $30 / 35$ & 85,71 \\
\hline & $\mathrm{T} 4$ & $131 / 192$ & 68,23 & $76 / 117$ & 64,96 & $55 / 75$ & 73,33 & $22 / 33$ & 66,67 & $80 / 124$ & 64,52 & $29 / 35$ & 82,86 \\
\hline & $\mathrm{T} 5$ & $127 / 192$ & 66,15 & $74 / 117$ & 63,25 & $53 / 75$ & 70,67 & $20 / 33$ & 60,61 & $78 / 124$ & 62,90 & $29 / 35$ & 82,86 \\
\hline & T6 & $125 / 192$ & 65,10 & $71 / 117$ & 60,68 & $54 / 75$ & 72,00 & $22 / 33$ & 66,67 & $80 / 124$ & 64,52 & $23 / 35$ & 65,71 \\
\hline & $\mathrm{T} 7$ & $121 / 192$ & 63,02 & $67 / 117$ & 57,26 & $54 / 75$ & 72,00 & $19 / 33$ & 57,58 & $81 / 124$ & 65,32 & $21 / 35$ & 60,00 \\
\hline & $\mathrm{T} 8$ & $121 / 192$ & 63,02 & $70 / 117$ & 59,83 & $51 / 75$ & 68,00 & $19 / 33$ & 57,58 & $78 / 124$ & 62,90 & $24 / 35$ & 68,57 \\
\hline & T9 & $119 / 192$ & 61,98 & $69 / 117$ & 58,97 & $50 / 75$ & 66,67 & $15 / 33$ & 45,45 & $81 / 124$ & 65,32 & $23 / 35$ & 65,71 \\
\hline & $\mathrm{T} 10$ & $117 / 192$ & 60,94 & $68 / 117$ & 58,12 & $49 / 75$ & 65,33 & $18 / 33$ & 54,55 & $78 / 124$ & 62,90 & $21 / 35$ & 60,00 \\
\hline & $\mathrm{T} 11$ & $122 / 192$ & 63,54 & $69 / 117$ & 58,97 & $53 / 75$ & 70,67 & $21 / 33$ & 63,64 & $80 / 124$ & 64,52 & $21 / 35$ & 60,00 \\
\hline & $\mathrm{T} 12$ & $123 / 192$ & 64,06 & $71 / 117$ & 60,68 & $52 / 75$ & 69,33 & $22 / 33$ & 66,67 & $80 / 124$ & 64,52 & $21 / 35$ & 60,00 \\
\hline \multirow{6}{*}{ 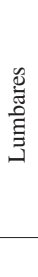 } & L1 & $153 / 192$ & 79,69, & $87 / 117$ & 74,36 & $66 / 75$ & 88,00 & $26 / 33$ & 78,79 & $102 / 124$ & 82,26 & $25 / 35$ & 71,43 \\
\hline & $\mathrm{L} 2$ & $147 / 192$ & 76,56 & $86 / 117$ & 73,50 & $61 / 75$ & 81,33 & $21 / 33$ & 63,64 & $102 / 124$ & 82,26 & $24 / 35$ & 68,57 \\
\hline & L3 & $143 / 192$ & 74,48 & $80 / 117$ & 68,38 & $63 / 75$ & 84,00 & $23 / 33$ & 69,70 & $98 / 124$ & 79,03 & $22 / 35$ & 62,86 \\
\hline & L4 & $147 / 192$ & 76,56 & $86 / 117$ & 73,50 & $61 / 75$ & 81,33 & $22 / 33$ & 66,67 & $99 / 124$ & 79,84 & $26 / 35$ & 74,29 \\
\hline & L5 & $151 / 192$ & 78,65 & $92 / 117$ & 78,63 & $59 / 75$ & 78,67 & $22 / 33$ & 66,67 & $103 / 124$ & 83,06 & $26 / 35$ & 74,29 \\
\hline & $\mathrm{S} 1$ & $140 / 192$ & 72,92 & $85 / 117$ & 72,65 & $55 / 75$ & 73,33 & $19 / 33$ & 57,58 & $98 / 124$ & 79,03 & $23 / 35$ & 65,71 \\
\hline
\end{tabular}

\section{Condiciones patológicas}

\section{Cuerpos vertebrales}

La Tabla 4 presenta las frecuencias y porcentajes de las manifestaciones de EVD registradas en toda la muestra y por grupos de sexo. Para toda la muestra, en los cuerpos vertebrales la manifestación más frecuente por FI y FV es la labiación $(79,10 \%$ y $48,73 \%$, respectivamente). Esta manifestación es la más observada en todas las regiones anatómicas (Tabla 4), pero en la mayoría de los casos se expresa de forma muy leve (Figura 4). Por otra parte, la manifestación más frecuentemente clasificada como código 3 y 4 son los osteofitos (Figura 4).

Entre los cuerpos vertebrales de individuos masculinos y femeninos la FI y FV de las manifestaciones varían ligeramente, siendo los osteofitos la manifestación que presentamayordesproporción deFIy FV porsexo: fue registrada con mayor frecuencia en individuos femeninos (FI: 65,74\%; FV: 36,65\%) que en individuos masculinos (FI: 53,61\%; FV: 27,59\%) (Tabla 4). Por regiones, los individuos de sexo femenino presentan mayor porcentaje de osteofitos en los cuerpos vertebrales de las regiones cervical y torácica, mientras que los masculinos exhiben mayor FI y FV de labiación y porosidad en la región lumbar (Tabla 4). Los porcentajes entre grupos de sexo fueron comparados con una prueba $\chi^{2}$ (la Tabla 5 incluye los resultados). No se encontraron diferencias significativas para la FI en los cuerpos vertebrales entre los grupos de sexo, salvo por la porosidad en la región lumbar. La FV revela que en todas las regiones los cuerpos vertebrales femeninos están significativamente más afectados por osteofitos, mientras que los masculinos por labiación y 
Tabla 4. Frecuencias de manifestaciones de EVD (Enfermedad Vertebral Degenerativa) por grupos de sexo.

Frequencies of VDD (Vertebral Degenerative Disease) manifestations by sex categories.

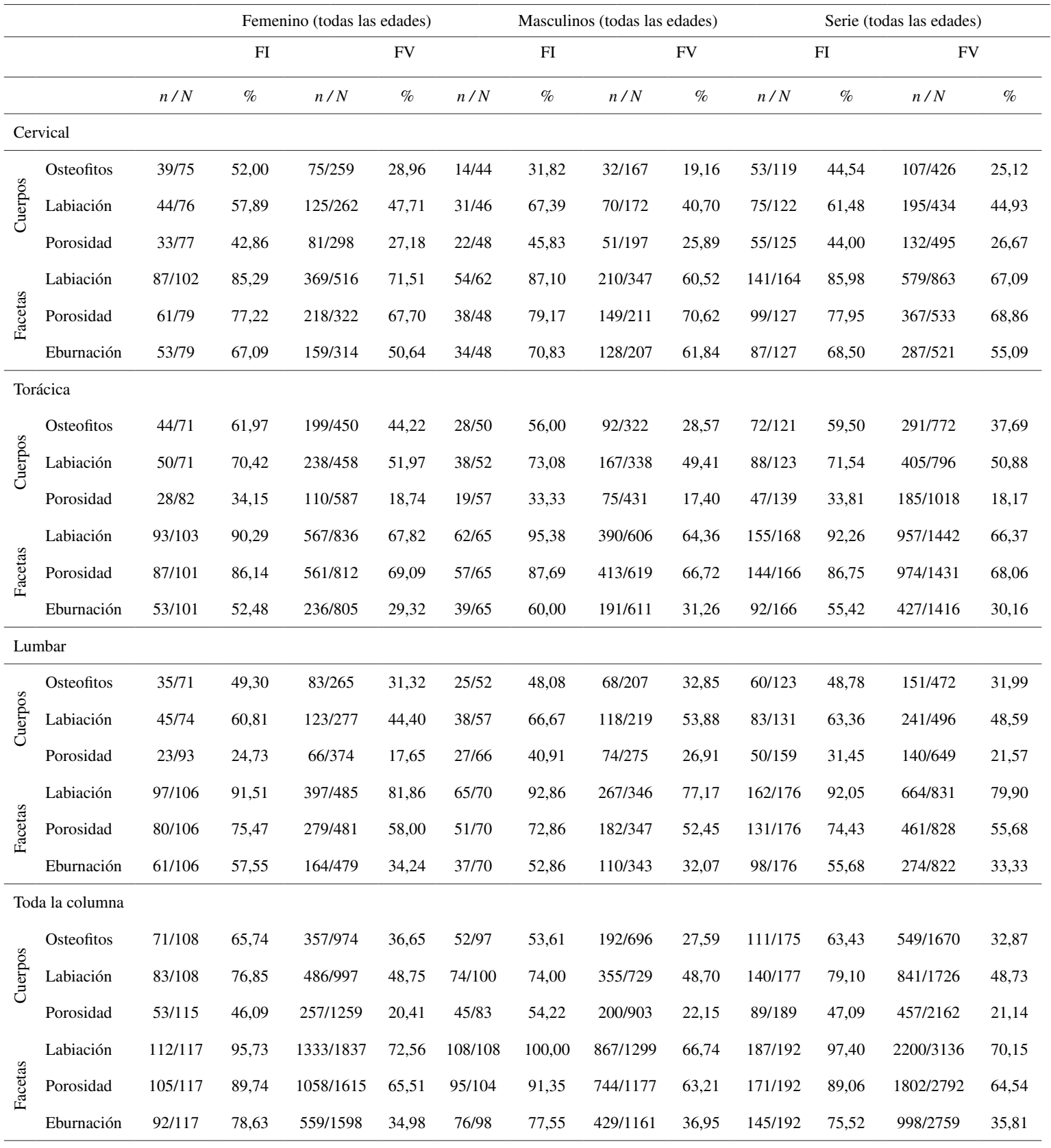


Porosidad

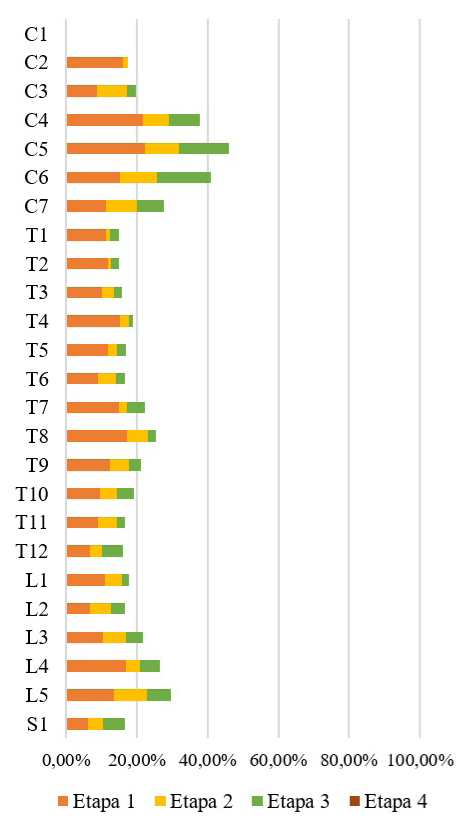

Labiación

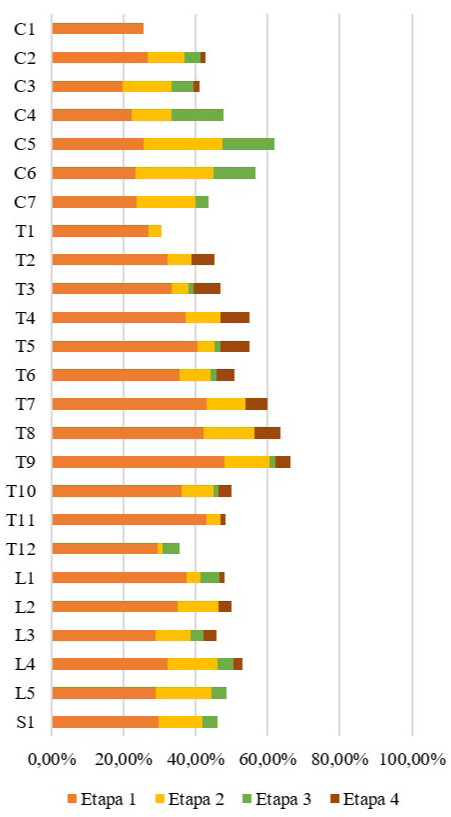

Osteofitos

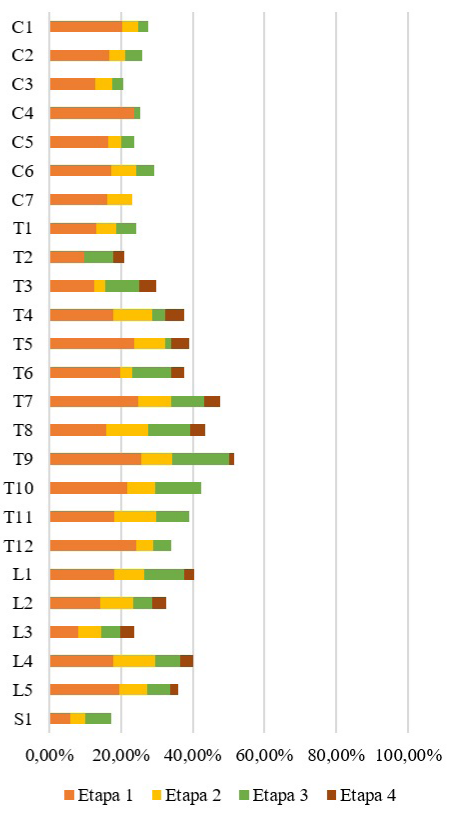

Figura 4. Manifestaciones de EVD (Enfermedad Vertebral Degenerativa) en los cuerpos vertebrales de la muestra por vertebra.

Manifestations of VDD (Vertebral Degenerative Disease) in the vertebral bodies of the sample by vertebra.

porosidad en la región lumbar, nótese que si bien se tomó el p-value de 0,05 como corte, la mayoría de valores se encuentran por debajo de 0,02 (Tabla 5).

En la Tabla 6 se observan las frecuencias de las manifestaciones por grupos de edad. En el grupo más joven (15-30 años) las manifestaciones de EVD en los cuerpos vertebrales son menos frecuentes que en individuos de mediana edad (30-45 años) y mayores (45+ años). Se encontraron diferencias significativas, principalmente entre la FV de los tres grupos de edad, a pesar de que se tomó el p-value de 0,05 como corte, la mayoría de valores se encuentran por debajo de 0,02 (Tabla 7). Para toda la columna vertebral, en los tres grupos la labiación es la condición más registrada en los cuerpos vertebrales, seguida de los osteofitos y la porosidad (Tabla 6). La frecuencia de las manifestaciones en la región torácica y lumbar aumenta progresivamente según la edad, salvo en la región cervical donde la FI de osteofitos es mayor en individuos jóvenes $(8 / 20,40,00 \%)$ que en individuos de mediana edad (28/74, 37,84\%); no obstante, la FV es más frecuente en adultos medios (56/249, 22,49\%) que en jóvenes $(14 / 77,18,18 \%)$ (Tabla 6).

\section{Facetas articulares}

Al contrastar la FI y FV para toda la columna vertebral, las facetas articulares presentan mayor afectación que los cuerpos vertebrales (Tabla 4). La labiación es la condición más registrada en las facetas articulares, afectando al 97,40\% de los individuos y al 70,15\% de las vértebras (Tabla 4). En contraste, la eburnación tiene las frecuencias FI $(75,52 \%)$ y FV $(35,81 \%)$ más bajas. Al igual que en los cuerpos vertebrales, en las facetas articulares se registra el código 1 (muy leve) como el más frecuente para todas las manifestaciones (Figura 5). La porosidad es la más registrada como moderada y severa, mientras que los casos muy severos de labiación tienden a localizarse en los segmentos C2-C4, T2-T5 y L4-S1 (Figura 5).

Al comparar frecuencias, porcentajes y localizaciones de las manifestaciones por regiones anatómicas, se observa que la eburnación y la porosidad son más frecuentes en las facetas cervicales; y la labiación es más prevalente en las facetas lumbares (Figura 5). Para todos los individuos la labiación presenta la mayor FI en toda la columna vertebral (Tabla 4). No obstante, la FV indica que la porosidad afecta mayormente a los individuos masculinos en la región cervical $(70,62 \%)$ y torácica $(66,72 \%)$. Como se observa en la Tabla 4 , la FI y FV de las manifestaciones son similares entre los sexos, salvo que los individuos masculinos tienen mayor FI de eburnación en la región torácica $(60,00 \%$ contra $52,48 \%$ ). No se hallaron diferencias significativas para la FI de las manifestaciones entre los grupos por sexo; sin embargo, las diferencias son significativas para la 
Tabla 5. Prueba $\boldsymbol{\chi}^{2}$ y Test de Fisher para manifestaciones en regiones vertebrales por grupos de sexo (valores significativos en negrita).

Chi-square $\left(\boldsymbol{\chi}^{2}\right)$ and $P$-values for manifestations in the vertebral regions by sex (significant values are in bold).

\begin{tabular}{|c|c|c|c|c|c|}
\hline & & \multicolumn{2}{|c|}{$\begin{array}{l}\text { Diferencia individuos } \\
\text { masculinos-femeninos FI }\end{array}$} & \multicolumn{2}{|c|}{$\begin{array}{l}\text { Diferencia individuos } \\
\text { masculinos-femeninos FV }\end{array}$} \\
\hline & & $X^{2}$ & $\mathrm{p}$ & $X^{2}$ & $\mathrm{p}$ \\
\hline \multicolumn{6}{|c|}{ Cervical } \\
\hline \multirow{3}{*}{ 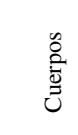 } & Osteofitos & 4,57258381 & 0,03248753 & 5,18007242 & 0,02284737 \\
\hline & Labiación & 1,09120275 & 0,29620487 & 2,06348656 & 0,15086464 \\
\hline & Porosidad & 0,10629252 & 0,74440519 & 0,10137379 & 0,75018721 \\
\hline \multirow{3}{*}{ 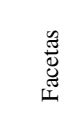 } & Labiación & 0,10392373 & 0,74717086 & 11,35578864 & 0,00075213 \\
\hline & Porosidad & 0,06616154 & 0,79700983 & 0,50482995 & 0,47738554 \\
\hline & Eburnación & 0,19405857 & 0,65955995 & 6.32387700 & 0,01191229 \\
\hline \multicolumn{6}{|c|}{ Torácica } \\
\hline \multirow{3}{*}{ 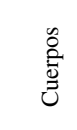 } & Osteofitos & 0,43421034 & 0,50993020 & 19,57583751 & 0,00000967 \\
\hline & Labiación & 0,10388309 & 0,74721862 & 0,50868706 & 0,47570777 \\
\hline & Porosidad & 0,00993133 & 0,92061737 & 0,29918032 & 0,58439674 \\
\hline \multirow{3}{*}{ 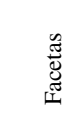 } & Labiación & 1,44807951 & 0,22883636 & 1,89138799 & 0,16904545 \\
\hline & Porosidad & 0,00993133 & 0,92061737 & 0,90621379 & 0,34112101 \\
\hline & Eburnación & 0,90637456 & 0,34107819 & 0,62291232 & 0,42996710 \\
\hline \multicolumn{6}{|c|}{ Lumbar } \\
\hline \multirow{3}{*}{ 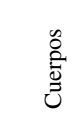 } & Osteof & 0,01784747 & 0,89372330 & 0,12495905 & 0,72371702 \\
\hline & Labiación & 0,47559721 & 0,49042340 & 4,39729080 & 0,03599607 \\
\hline & Porosidad & 4,68675482 & 0,03039600 & 8,03555239 & 0,00458680 \\
\hline \multirow{3}{*}{ 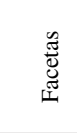 } & Labiación & 0,10458411 & 0,74639637 & 2,76386570 & 0,09641501 \\
\hline & Porosidad & 0,15143575 & 0,69716706 & 2,52024034 & 0,11239345 \\
\hline & Eburnación & 0,37579117 & 0,53986438 & 0,42276487 & 0,51556064 \\
\hline \multicolumn{6}{|c|}{ Columna } \\
\hline \multirow{3}{*}{ 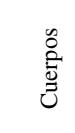 } & Osteofitos & 3,1 & 70 & 15,12209486 & 0,00010078 \\
\hline & Labiación & 0,22817664 & 0,63287913 & 0,00041121 & 0,98382128 \\
\hline & Porosidad & 1,27463730 & 0,25889852 & 0,94997411 & 0,32972589 \\
\hline \multirow{3}{*}{ 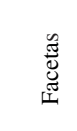 } & Labiación & & 0,06070000 & 12,31086773 & 0,00045033 \\
\hline & Porosidad & 0,16443452 & 0,68510615 & 1,57274592 & 0,20980869 \\
\hline & Eburnación & 0,03651428 & 0,84845735 & 1,13494704 & 0,28672258 \\
\hline
\end{tabular}


Tabla 6. Frecuencias de manifestaciones de EVD (Enfermedad Vertebral Degenerativa) por grupos de edad.

Frequency of VDD (Vertebral Degenerative Disease) features by age categories.

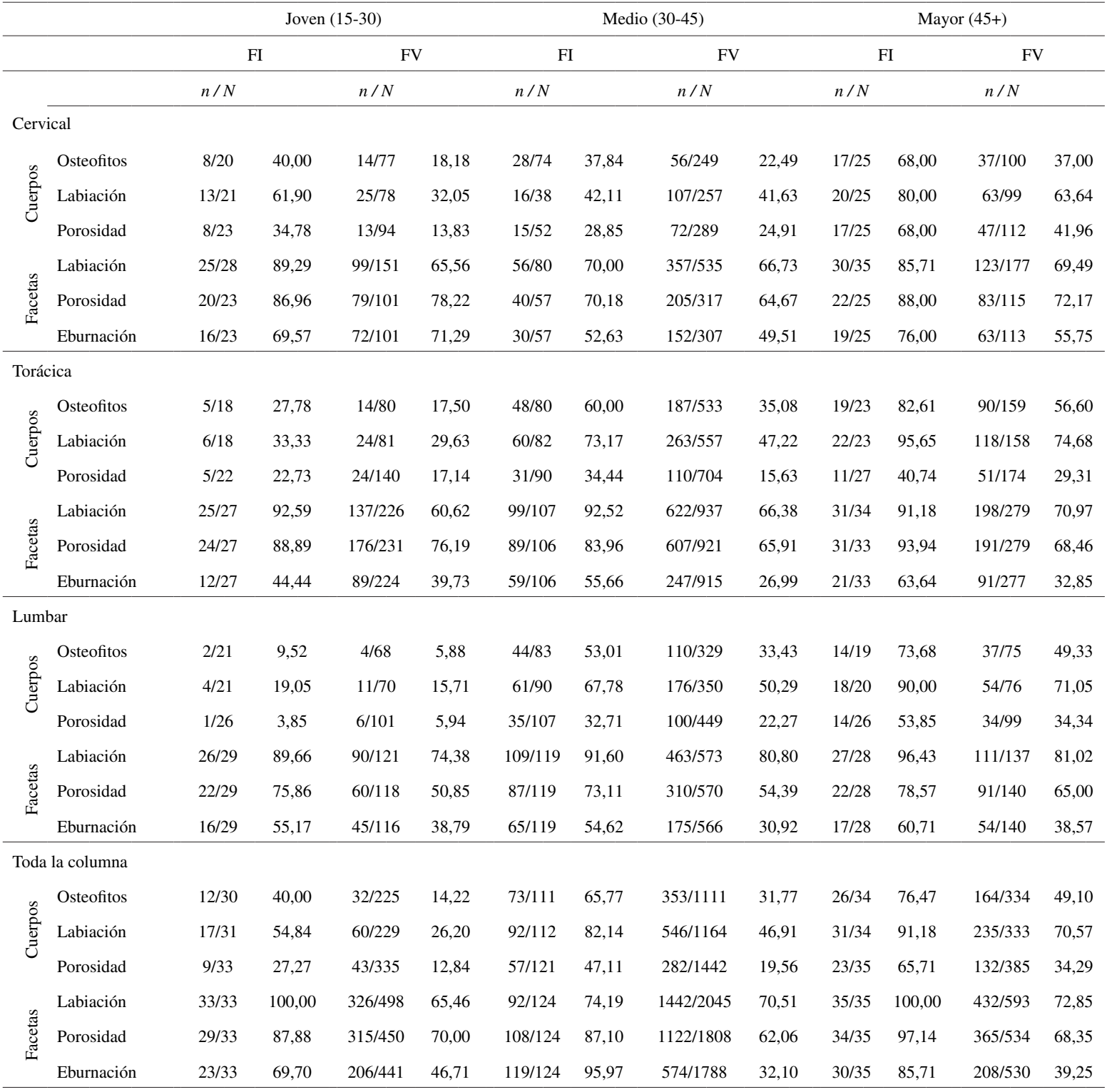




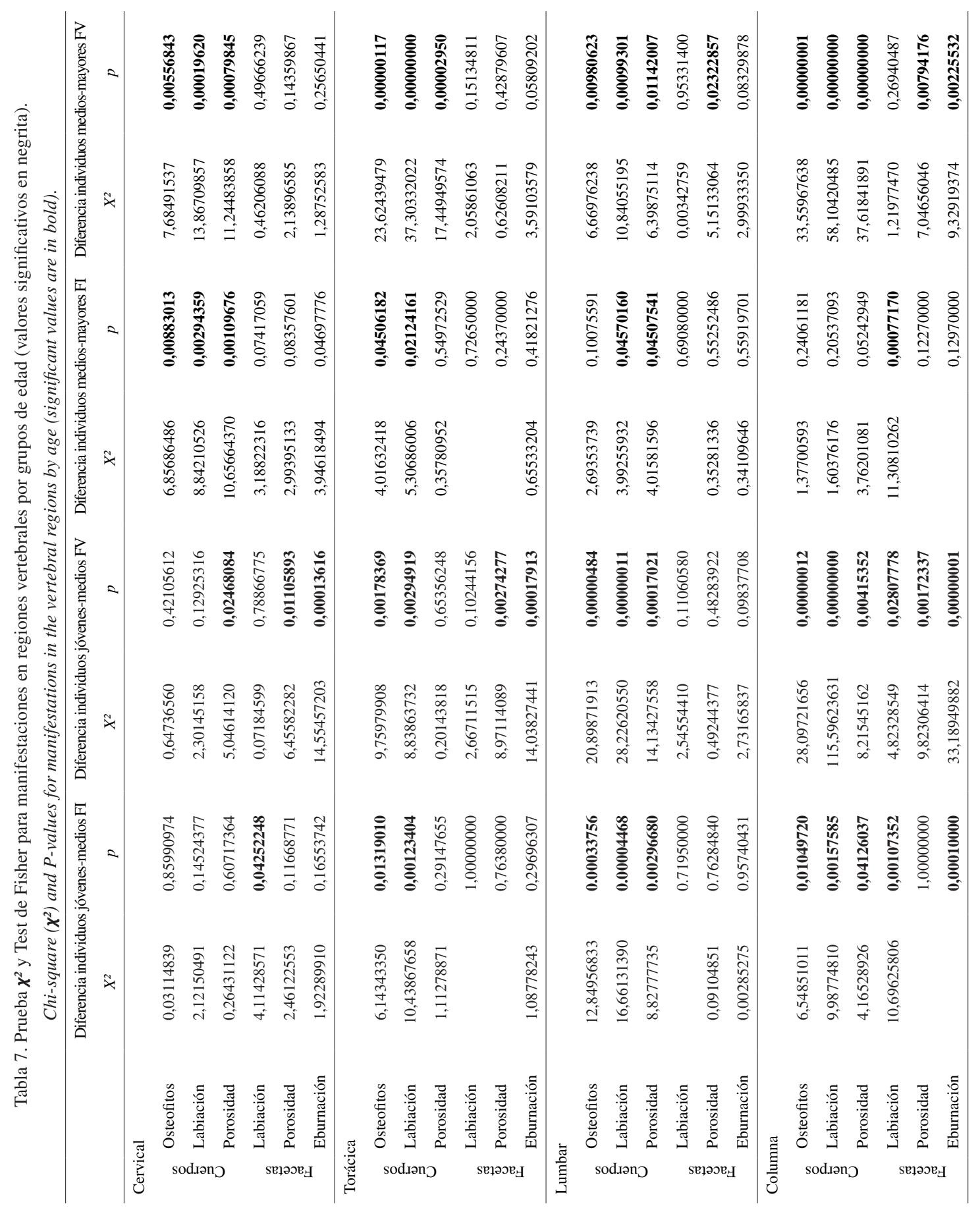


Porosidad

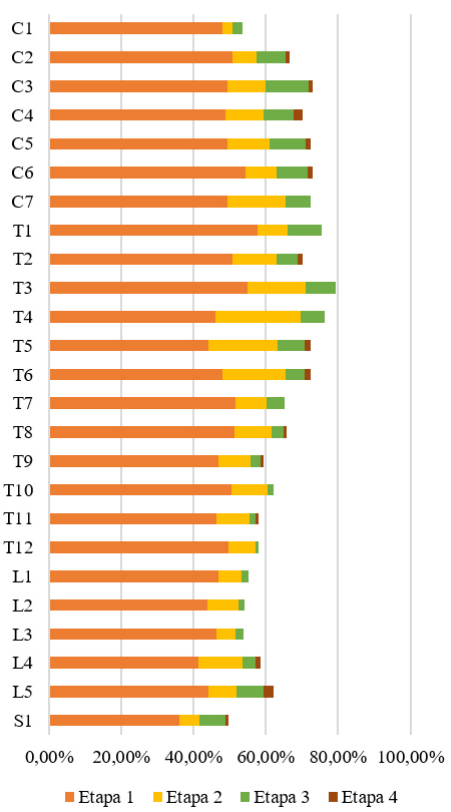

Labiación

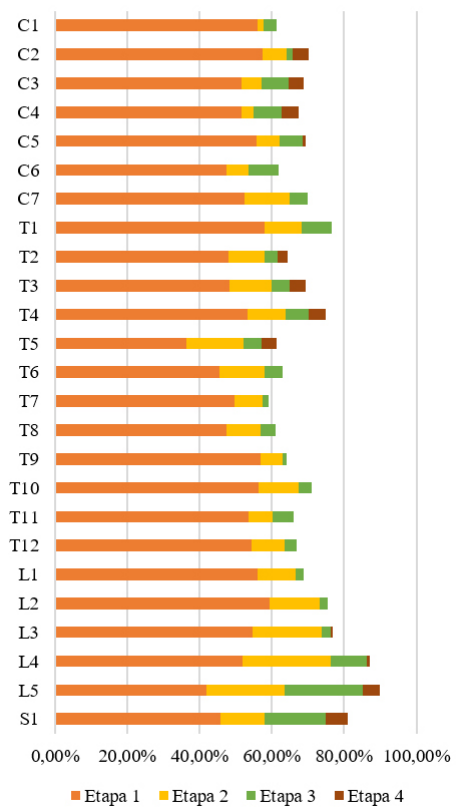

Eburnación

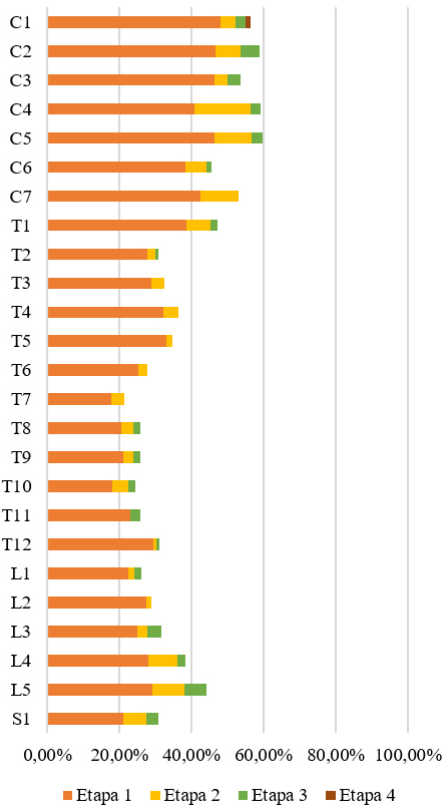

Figura 5. Manifestaciones de EAD (Enfermedad Articular Degenerativa) en las facetas articulares de la muestra por vertebra.

Manifestations of DJD (Degenerative Joint Disease) in the apophyseal joints of the sample by vertebra.

$\mathrm{FV}$, principalmente en las facetas articulares de la región cervical; como se puede observar el p-value de 0,05 fue tomado como corte, pero la mayoría de los valores se encuentran por debajo de 0,02 (Tabla 5).

Para toda la columna vertebral, tanto la porosidad como la eburnación en las facetas articulares aumenta según la edad, siendo los individuos mayores los más afectados (Tabla 6). Al hacer el cálculo por FI, la prueba $\chi^{2}$ revela diferencias significativas de porosidad y eburnación entre individuos jóvenes y medios; y de porosidad entre los individuos medios y mayores, aunque se tomó el p-value de 0,05 como corte, la mayoría de los valores se encuentran por debajo de 0,02 (Tabla 7). Los valores son significativos al comparar la FV de todos los grupos de edad (Tabla 7). Por regiones anatómicas, las facetas cervicales de los individuos jóvenes se ven más afectadas por eburnación y labiación que en otros grupos (Tabla 6), siendo significativamente más frecuentes; como se puede observar, aunque se tomó el p-value de 0,05 como corte, la mayoría de los valores son inferiores a 0,02 (Tabla 7).

\section{Método de diagnóstico}

El registro de las expresiones leves (código 1) de las manifestaciones puede ser una dificultad; entre más incipientes sean, más difícil es codificarlas, lo cual puede conducir a una sobreestimación de la EAD (Waldron y Rogers 1991; Zampetti et al. 2016). Varios autores han resaltado esta situación y algunos, como Zampetti et al. (2016), sugieren que la única manera de reducir los errores es sólo codificando las expresiones obvias de la EAD (códigos 2, 3 y 4). En consecuencia, para el diagnóstico de la EVD en la presente investigación solo tiene en consideración los códigos 2, 3 y 4 de las manifestaciones.

La Tabla 8 presenta las frecuencias de EVD en las regiones anatómicas de la columna vertebral de toda la muestra y por grupos de sexo. El método diagnostica como positivo a $75,56 \%$ de los individuos y a $27,52 \%$ de las vértebras. La región anatómica con mayor afectación en toda la muestra es la región lumbar, la cual presenta $59,22 \%$ FI y $35,64 \%$ FV. Para toda la columna vertebral, ambos sexos exhiben frecuencias similares: $76,07 \%$ FI y $28,41 \%$ FV en femeninos; $77,33 \%$ FI y $26,25 \%$ FV en masculinos (Tabla 8). Por regiones anatómicas, el sexo masculino presenta mayor FI en las tres regiones. En lo que respecta a la FV, es más elevada en las regiones cervical y torácica de individuos femeninos; y en la región lumbar de los individuos masculinos (Tabla 8). No se hallaron diferencias significativas entre los grupos de sexo (Tabla 9). 
Tabla 8. EVD (Enfermedad Vertebral Degenerativa) en regiones anatómicas de la columna vertebral por grupos de sexo.

$V D D$ (Vertebral Degenerative Disease) in anatomical regions of the vertebral column by sex categories.

\begin{tabular}{lccccccccccccc}
\hline & \multicolumn{3}{c}{ Femeninos (todas las edades) } & \multicolumn{3}{c}{ Masculinos (todas las edades) } & \multicolumn{3}{c}{ Serie (todas las edades) } \\
\hline & \multicolumn{2}{c}{ FI } & \multicolumn{2}{c}{ FV } & & \multicolumn{2}{c}{ FI } & & FV & & FI & & FV \\
\hline & $n / N$ & $\%$ & $n / N$ & $\%$ & $n / N$ & $\%$ & $n / N$ & $\%$ & $n / N$ & $\%$ & $n / N$ & $\%$ \\
\hline Cervical & $44 / 102$ & 43,14 & $124 / 521$ & 23,80 & $29 / 62$ & 46,77 & $72 / 349$ & 20,63 & $73 / 164$ & 44,51 & $196 / 870$ & 22,53 \\
Torácica & $57 / 104$ & 54,81 & $238 / 863$ & 27,58 & $44 / 65$ & 67,69 & $145 / 627$ & 23,13 & $101 / 169$ & 59,76 & $383 / 1490$ & 25,70 \\
Lumbar & $61 / 107$ & 57,01 & $175 / 506$ & 34,58 & $45 / 72$ & 62,50 & $134 / 361$ & 37,12 & $106 / 179$ & 59,22 & $309 / 867$ & 35,64 \\
Columna & $89 / 117$ & 76,07 & $537 / 1890$ & 28,41 & $58 / 75$ & 77,33 & $351 / 1337$ & 26,25 & $147 / 192$ & 76,56 & $888 / 3227$ & 27,52 \\
\hline
\end{tabular}

Tabla 9. Prueba $\boldsymbol{\chi}^{2}$ y Test de Fisher para EVD (Enfermedad Vertebral Degenerativa) en regiones vertebrales por grupos de sexo.

Chi-square $\left(\boldsymbol{\chi}^{2}\right)$ and $P$-values for VDD (Vertebral Degenerative Disease) in the vertebral regions by sex categories.

\begin{tabular}{lcccc}
\hline & \multicolumn{2}{c}{$\begin{array}{c}\text { Diferencia individuos } \\
\text { masculinos-femeninos FI }\end{array}$} & \multicolumn{2}{c}{$\begin{array}{c}\text { Diferencia individuos } \\
\text { masculinos-femeninos FV }\end{array}$} \\
\hline Cervical & 0,20651110 & 0,64951582 & 1,20333924 & 0,27265529 \\
Torácica & 2,76150626 & 0,09655729 & 3,76939103 & 0,05219863 \\
Lumbar & 0,53726527 & 0,46356830 & 0,58985201 & 0,44247635 \\
Columna & 0,04075400 & 0,84001351 & 1,83152864 & 0,17594710 \\
\hline
\end{tabular}

Las frecuencias de EVD en las regiones anatómicas de la columna vertebral por grupos de edad se presentan en la Tabla 10. Como tendencia general se observa un aumento progresivo tanto de la FI como de la $\mathrm{FV}$ en los grupos de edad, siendo los individuos mayores (45+ años) los más afectados por la patología. En los individuos jóvenes (15-30 años) la EVD afectó al $39,39 \%$ de las columnas y al $10,51 \%$ de las vértebras; la región anatómica más afectada es la región lumbar con $26,67 \%$ FI y $17,46 \%$ FV. Para los individuos de edad mediana (30-45 años) aumentan las frecuencias para toda la columna vertebral: $82,26 \%$ FI y $28,86 \%$ $\mathrm{FV}$; la región más afectada es la región lumbar con $63,33 \%$ FI y $36,68 \%$ FV. En individuos mayores (45+ años) las frecuencias alcanzan 91,43\% FI y 43,92\% FV; a diferencia de los otros grupos de edad, la FI es mayor en la región torácica $(70,59 \%)$ y la $\mathrm{FV}$, en la región lumbar $(47,22 \%)$ (Tabla 10).

La Tabla 11 presenta la comparación de la prevalencia de EVD, región por región, entre los grupos de edad. Si los cálculos son hechos por FI, entre adultos jóvenes y medios hay diferencias en las regiones torácica y lumbar; en contraste, entre los adultos medios y mayores sólo hay diferencia en la región cervical. Sin embargo, al hacer la comparación por FV, se observan diferencias entre todas las regiones de los tres grupos, una vez más, se puede notar que a pesar de que se tomó el p-value de 0,05 como corte, la mayoría de los valores se encuentran por debajo de 0,02 (Tabla 11).

Tabla 10. EVD (Enfermedad Vertebral Degenerativa en regiones anatómicas de la columna vertebral por grupos de edad.

VDD (Vertebral Degenerative Disease) in anatomical regions of the vertebral column by age categories.

\begin{tabular}{|c|c|c|c|c|c|c|c|c|c|c|c|c|}
\hline & \multicolumn{4}{|c|}{ Jóvenes $(15$ - 30) } & \multicolumn{4}{|c|}{ Medio 30-45 } & \multicolumn{4}{|c|}{ Mayor 45+ } \\
\hline & \multicolumn{2}{|c|}{ FI } & \multicolumn{2}{|c|}{ FV } & \multicolumn{2}{|c|}{ FI } & \multicolumn{2}{|c|}{ FV } & \multicolumn{2}{|c|}{ FI } & \multicolumn{2}{|c|}{$\mathrm{FV}$} \\
\hline & $n / N$ & $\%$ & $n / N$ & $\%$ & $n / N$ & $\%$ & $n / N$ & $\%$ & $n / N$ & $\%$ & $n / N$ & $\%$ \\
\hline Cervical & $8 / 28$ & 28,57 & $15 / 151$ & 9,93 & $41 / 101$ & 40,59 & $107 / 541$ & 19,78 & $24 / 35$ & 68,57 & $74 / 178$ & 41,57 \\
\hline Torácica & $7 / 28$ & 25,00 & $17 / 237$ & 7,17 & $70 / 107$ & 65,42 & $237 / 958$ & 24,74 & $24 / 34$ & 70,59 & $129 / 295$ & 43,73 \\
\hline Lumbar & $8 / 30$ & 26,67 & $22 / 126$ & 17,46 & $76 / 120$ & 63,33 & $219 / 597$ & 36,68 & $22 / 29$ & 63,33 & $68 / 144$ & 47,22 \\
\hline Columna & $13 / 33$ & 39,39 & $54 / 514$ & 10,51 & $102 / 124$ & 82,26 & $563 / 2096$ & 26,86 & $32 / 35$ & 91,43 & $271 / 617$ & 43,92 \\
\hline
\end{tabular}

Tabla 11. Prueba $\boldsymbol{\chi}^{2}$ y Test de Fisher para EVD (Enfermedad Vertebral Degenerativa) en regiones vertebrales por grupos de edad (valores significativos están en negrita)

Chi-square $\left(\chi^{2}\right)$ and P-values for VDD (Vertebral Degenerative Disease) in the vertebral regions by age groups (significant values are in bold)

\begin{tabular}{ccccccccrrr}
\hline & \multicolumn{2}{c}{$\begin{array}{c}\text { Diferencia individuos jóvenes- } \\
\text { medios FI }\end{array}$} & \multicolumn{2}{c}{$\begin{array}{c}\text { Diferencia individuos } \\
\text { jóvenes-medios FV }\end{array}$} & \multicolumn{2}{c}{$\begin{array}{c}\text { Diferencia individuos medios- } \\
\text { mayores FI }\end{array}$} & $\begin{array}{c}\text { Diferencia individuos medios- } \\
\text { mayores FV }\end{array}$ \\
\hline & \multicolumn{2}{c}{$X^{2}$} & $p$ & $X^{2}$ & $p$ & $X^{2}$ & $p$ & $X^{2}$ & $p$ \\
\hline Cervical & 1.34518517 & 0,24612162 & 7,87816973 & $\mathbf{0 , 0 0 5 0 0 3 5 1}$ & 8,15399363 & $\mathbf{0 , 0 0 4 2 9 6 6 4}$ & 33,77480758 & $\mathbf{0 , 0 0 0 0 0 0 0 1}$ \\
Torácica & 14.79658615 & $\mathbf{0 , 0 0 0 1 1 9 7 5}$ & 35,02727461 & $\mathbf{0 , 0 0 0 0 0 0 0 0}$ & 0,31006047 & 0,57764308 & 39,33448590 & $\mathbf{0 , 0 0 0 0 0 0 0 0}$ \\
Lumbar & 15.53677396 & $\mathbf{0 , 0 0 0 0 8 0 9 2}$ & 17,30078966 & $\mathbf{0 , 0 0 0 0 3 1 9 1}$ & 1,62848496 & 0,20191273 & 5,43000251 & $\mathbf{0 , 0 1 9 7 9 3 6 5}$ \\
Columna & 24.43858602 & $\mathbf{0 , 0 0 0 0 0 0 7 7}$ & 61,16396009 & $\mathbf{0 , 0 0 0 0 0 0 0 0}$ & 1,73232244 & 0,18811457 & 65,17314840 & $\mathbf{0 , 0 0 0 0 0 0 0 0}$ \\
\hline
\end{tabular}




\section{Discusión y Conclusión}

La diferencia anatómica entre las articulaciones de la columna vertebral, los cuerpos vertebrales y las facetas articulares también implica una diferencia en la prevalencia y distribución de las manifestaciones asociadas a la EVD (Bridges 1994; Knüsel et al. 1997; Resnick 2002; Sofaer 2000). El deterioro óseo en los cuerpos vertebrales es asociado principalmente a los puntos de curvatura de la columna vertebral: C5, C6, T7, T8, L3 y L4 (Sofaer 2000), aunque en ciertos casos ha sido asociado a determinadas actividades (Bridges 1994; Merbs 1983). En el presente estudio las manifestaciones en los cuerpos vertebrales tienden a concentrarse en algunos puntos de curvatura de la columna vertebral (Figura 2), lo cual indica que efectivamente las estructuras se vieron afectadas tanto por la bipedación como por la actividad física. Por otra parte, la afectación en las facetas articulares se relaciona más a actividad física, pues su patrón suele ser distinto y las frecuencias de afectación más elevadas que en los cuerpos vertebrales (Bridges 1994; Sofaer 2000). En la serie del Globo B se halló que los cambios óseos son más frecuentes en las facetas articulares que en los cuerpos vertebrales, lo cual se podría asociar a un estilo de vida más extenuante. No obstante, estos cambios se expresaban en su mayoría de manera leve, lo que no excluye la posibilidad que factores como la edad y la bipedación, entre otros, pudieran afectar estas estructuras. Por lo tanto, es mejor observar los resultados a través de un método de diagnóstico.

El diagnóstico de EVD permite proponer tres hipótesis sobre la población inhumada en el Globo B del Cementerio Central de Bogotá: la alta prevalencia de EVD estaría relacionada a un estilo de vida con habitual actividad física; la alta prevalencia en la población joven (15 - 30 años) sería consecuencia de actividades laborales desde edad temprana; y, la prevalencia similar, pero con un patrón distinto de distribución de EVD en ambos sexos, sería consecuencia de diferentes tipos de estrés, por lo que ciertas actividades y oficios pudieron ser exclusivos de uno u otro sexo. En la muestra del Globo B no es posible especificar qué actividades ejercieron los individuos, menos asociar actividades específicas a uno u otro sexo, puesto que el patrón no es lo suficientemente marcado. Para esto es menester analizar la totalidad del material esquelético disponible, ya que ciertas actividades afectan de manera inespecífica y distinta cada articulación (Jurmain 1980, 1991).

Con todo, la información histórica respalda los tres principales resultados de esta investigación. Primeramente, la alta prevalencia de EVD está relacionada a un estilo de vida con habitual actividad física. El trabajo manual fue una característica determinante de la clase media-baja bogotana de los siglos XIX y XX que, según los censos de Bogotá de 1859 y 1945 , eran esencialmente agricultores, obreros, peones, jornaleros, empleadas domésticas, entre otros (López-Uribe 2011; Tovar 1995). Es decir, eran trabajadores no calificados y probablemente, mal alimentados (Castaño 2016; Castiblanco 2016), los cuales aportaban su esfuerzo físico y efectuaban las tareas más pesadas (López 1939). Son escasas las descripciones sobre las actividades específicas que efectuaba cada grupo de trabajadores, es decir, si frecuentemente se doblaban, se acuclillaban o si levantaban objetos pesados (o si los llevaban sobre la espalda o la cabeza). Sumado al hecho de que en la colección el patrón de EVD no es muy marcado, atribuir ciertas ocupaciones y actividades a los individuos no es posible. Con todo, la Tabla 12 presenta los principales oficios reportados para Bogotá en el censo de Colombia de 1859.

Tabla 12. Principales oficios en Bogotá según el censo de 1956 (Tovar 1995:70-73).

Main occupations in Bogotá, according to the 1956 census (Tovar 1995:70-73).

\begin{tabular}{lcc}
\hline Principales oficios & $\mathrm{n}$ & $\%$ \\
\hline Agricultores & 23.780 & $31,43 \%$ \\
Infantes sin oficio & 21.405 & $28,29 \%$ \\
Admón. doméstica & 10.984 & $14,52 \%$ \\
Servicio doméstico & 7.311 & $9,66 \%$ \\
Artesanos & 6.037 & $7,98 \%$ \\
Otros (varios) & 2.976 & $3,93 \%$ \\
Estudiantes & 1.889 & $2,50 \%$ \\
Comerciantes & 1.271 & $1,68 \%$ \\
Total & 75.653 & \\
\hline
\end{tabular}

Otro punto es la variación del patrón de EVD según el sexo y su relación con actividades diferenciadas. Para mitad del siglo XIX, en Bogotá el oficio más usual era el de agricultor, seguido de la administración doméstica y el servicio doméstico, respectivamente (Tabla 12). Estas dos últimas eran actividades exclusivamente femeninas (López-Uribe 2011). En el siguiente siglo, la industrialización llegó a Bogotá y transformó la ciudad. La industria creció y las fábricas cada vez demandaban más mano de obra, generalmente hombres. Según el Censo Industrial de Colombia de 1945, el sector industrial de Bogotá tenía tres veces más obreros que obreras, y esta diferencia era mayor en las ramas del Gobierno distrital. Es decir, para esta época mucha de la población femenina seguía dedicándose a labores domésticas, mientras otro porcentaje considerable pasó a trabajar en industrias. Aunque las industrias solían contratar más hombres que mujeres, ciertas empresas tendían a contratar más mujeres que hombres en oficios 
no calificados, posiblemente porque era la mano de obra más barata (López-Uribe 2011). En consecuencia, tanto mujeres como hombres llevaron a cabo trabajos físicamente demandantes, pero en algunos casos los trabajos diferían según el sexo. Esto se observa en los resultados osteológicos: alta prevalencia de EVD en ambos sexos, pero patrón de distribución distinto en cada uno.

Esta variación también se documenta en una muestra precolombina Muisca del actual territorio colombiano, cuyo análisis aplicó la misma metodología de registro que este estudio (Rojas-Sepúlveda et al. 2008). La comparación con esta colección es relevante ya que los Muiscas fueron cazadores-recolectores y plantadores que habitaron entre el 700 y el 1600 d.C. en el altiplano cundiboyacense colombiano (Rodríguez 1999); área en la que hoy día se sitúa la ciudad de Bogotá (Figura 1). En específico, la serie Muisca de Soacha data entre los siglos XI y XIII (Rodríguez 1994, 1999). Por otro lado, la población del Globo B es mestiza con predominante influencia indígena, y fue inhumada entre los siglos XIX y XX; cuando Bogotá estaba en transición hacia la industrialización (LópezBejarano 2019; López-Uribe 2011). La información histórica señala que durante este período el trabajo era extenuante, las condiciones laborales eran malas, los salarios eran bajos y las jornadas, extensas; además eran frecuentes los accidentes laborales y la precariedad de las condiciones higiénicas (López-Uribe 2011). Por lo tanto, los individuos de las dos series experimentaron condiciones de vida disímiles, a pesar de haber habitado el mismo territorio (Figura 1) en períodos distintos. La Tabla 13 presenta la prevalencia de EVD por regiones de la columna vertebral y por grupos de sexo en la muestra del Globo B y en la mencionada serie Muisca de Soacha.

Tabla 13. EVD (Enfermedad Vertebral Degenerativa) en regiones anatómicas de la columna vertebral en las muestras del Cementerio Centra de Bogotá y Muisca de Soacha (Rojas-Sepúlveda et al. 2008).

$V D D$ (Vertebral Degenerative Disease) in anatomical regions of the vertebral column found in the samples of the Central Cemetery of Bogotá and Soacha Muisca (Rojas-Sepúlveda et al. 2008).

\begin{tabular}{ccccccc}
\hline & \multicolumn{3}{c}{ Cementerio Central } & \multicolumn{3}{c}{ Soacha Muisca } \\
& Femenino & Masculino & Serie & Femenino & Masculino & Serie \\
\hline Cervical & 43,1 & 46,7 & 44,5 & 68,2 & 56,3 & 63,3 \\
Torácica & 54,8 & 67,6 & 59,7 & 60,0 & 64,5 & 62,0 \\
Lumbar & 57,0 & 62,5 & 59,2 & 69,8 & 59,4 & 64,9 \\
Columna & 76,0 & 77,3 & 76,5 & 89,1 & 76,5 & 83,1 \\
\hline
\end{tabular}

Al comparar el patrón de distribución de EVD en las dos muestras, es evidente que la afectación es distinta tanto a nivel inter-poblacional como intrapoblacional (Tabla 13). Esto se explicaría en que el perfil paleoepidemiológico de la EAD varía en función del estilo de vida de cada población (Rojas-Sepúlveda 2009) así como de los grupos que la conforman. El estilo de vida hace referencia a las relaciones con el ambiente, las estrategias de subsistencia y las circunstancias históricas de cada sociedad (Larsen 1997; RojasSepúlveda 2009). En consecuencia, cada población experimenta condiciones específicas que afectan de manera distinta a los individuos (estrés). El hecho que dos poblaciones tengan un perfil paleoepidemiológico y un patrón de distribución de EVD distinto reafirma este punto y, asimismo, refuta el planteamiento de que el factor determinante de los cambios degenerativos en la columna vertebral es la bipedación (Knüsel et al. 1997). Efectivamente, la postura bípeda genera estrés sobre los puntos de curvatura de la columna vertebral (vértebras C5, C6, T7, T8, L3 y L4), pero es el estrés biomecánico y otros factores sistémicos y locales los que marcan el desarrollo y severidad de la EVD (Bridges 1994; RojasSepúlveda 2009).

Finalmente, se halló una alta prevalencia de EVD en la población joven del Globo B, lo cual se relaciona con el inicio de actividades físicas repetitivas (como las de un oficio o un trabajo) desde temprana edad. Sin duda, el trabajo infantil y juvenil era una constante en la Bogotá de los siglos XIX y XX. Si bien en los censos se les reportaban como sin oficio (Tabla 13), los niños pobres embolaban zapatos, voceaban la prensa y trabajaban en fábricas o en negocios familiares, entre otros (López-Uribe 2011; Martínez 1991; Muñoz 1990). Con respecto al trabajo en las fábricas, en estas se prefería contratar "muchachos" (hombres jóvenes o adolescentes) a mujeres, siendo incluso mayor su salario (López-Uribe 2011). Coy (2019), en su estudio de los individuos infantes y adolescentes de la colección, también llega a la misma conclusión sobre la temprana inserción laboral de esta población; y la confirma con la revisión del Libro Necrológico correspondiente a 1902, las fotografías del fondo fotográfico Daniel Rodríguez y las publicadas por Sánchez Salcedo en 2014 (Coy 2019), cuyos registros referencian los oficios de los que hoy consideraríamos niños. En efecto, el trabajo hizo parte de la vida de los bogotanos desde muy temprana edad.

Agradecimientos: Agradecemos profundamente a las arqueólogas Sandra Mendoza y Katherine Mejía del Instituto Distrital de Patrimonio Cultural de Bogotá (IDPC) por gestionar lo necesario para el buen manejo de la colección. También, agradecemos a los editores de Chungara Revista de Antropología Chilena y a los evaluadores por sus oportunos comentarios. Esta investigación fue financiada por la Facultad de Ciencias Humanas de la Universidad Nacional de Colombia a través de la Beca Orlando Fals Borda. 


\section{Referencias Citadas}

Andrade, P., D. Salazar, J. Urrea y V. Castro 2014. Modos de vida de los cazadores-recolectores de la costa arreica del Norte Grande de Chile: una aproximación bioarqueológica a las poblaciones prehistóricas de Taltal. Chungara Revista de Antropología Chilena 46 (3):467-491

Bridges, P. 1991. Degenerative joint disease in hunter-gatherers and agriculturalists from the southeastern United States. American Journal of Physical Anthropology 85 (4):379-391.

Bridges, P. 1992. Prehistoric arthritis in the Americas. Annual Review of Anthropology, 21 (1):67-91.

Bridges, P. 1994. Vertebral arthritis and physical activities in the prehistoric southeastern United States. American Journal of Physical Anthropology 93 (1):83-93.

Brooks, S. y J. Suchey 1990. Skeletal age determination based on the os pubis: a comparison of the Acsádi-Nemeskéri and SucheyBrooks methods. Human Evolution 5 (3):227-238.

Buikstra, J. y D. Ubelaker 1994. Standards for data collection from human skeletal remains. Arkansas Archeological Survey Research Series No. 44, Fayetteville.

Calvo, O. 1998. El Cementerio Central: Bogotá, la Vida Urbana y la Muerte. Observatorio de Cultura Urbana, Bogotá.

Castaño, D. 2017. La Historia en los Dientes: una Aproximación Bioarqueológica con Enfoque en Antropología Dental, a las Condiciones de Vida de la Población Inhumada en el Cementerio Central de Bogotá, Globo B. Finales del Siglo XIX y Siglo $X X$. Monografía de pregrado. Departamento de Antropología, Universidad Nacional de Colombia, Bogotá.

Castiblanco, J. 2017. Estudio para la Estimación de la Estatura por Osteometría en los Restos Óseos de la Colección Osteológica del Cementerio Central de Bogotá de Finales del Siglo XIX y Principios del Siglo XX. Monografía de pregrado. Departamento de Antropología, Universidad Nacional de Colombia, Bogotá.

Cooper, C., T. McAlindon, D. Coggon, P. Egger y P. Dieppe 1994. Occupational activity and osteoarthritis of the knee. Annals of Rheumatic Diseases 53:90-93.

Coy, L. 2019. Condiciones de Vida de los Individuos Infantiles de Finales del Siglo XIX y Mediados del Siglo XX, a Partir de los Restos Óseos del Globo B del Cementerio Central-Bogotá. Tesis para optar al grado de Magister en Antropología, Departamento de Antropología, Universidad Nacional de Colombia, Bogotá.

Dieppe, P. 1995. The classification and diagnosis of osteoarthritis. En Osteoarthritic Disorders, editado por K. Kuettner y V. Goldberg, pp. 5-12. American Academy of Orthopaedic Surgeons, Rosemont.

Doherty, M., H. Bijlsma, N. Arden, N. Dalbeth y D. Hunter (eds.) 2016. Oxford Textbook of Osteoarthritis and Crystal Arthropathy. Oxford University Press, Oxford.

Gellhorn, A., J. Katz y P. Suri 2013. Osteoarthritis of the spine: the facet joints. Nature Reviews Rheumatology 9 (4):216-224.

Giannotti, S. 2016. Marcadores de estrés ocupacional en poblaciones históricas del norte de Mendoza (s. XVI-XVII): primeros resultados exploratorios. Comechingonia: Revista de Arqueología 20 (1):81-110.

Hunter, D. y D. Felson 2006. Osteoarthritis. BMJ 332 (7542):639642.
Isçan, M., S. Loth y R. Wright 1984. Age estimation from the rib by phase analysis: White males. Journal of Forensic Science 29:10941104.

Isçan, M., S. Loth y R. Wright 1985. Age estimation from the rib by phase analysis: White females. Journal of Forensic Science 30:853863.

Jurmain, R. 1977. Stress and the etiology of osteoarthritis. American Journal of Physical Anthropology 46 (2):353-366.

Jurmain, R. 1980. The pattern of involvement of appendicular degenerative joint disease. American Journal of Physical Anthropology 53 (1):143-150.

Jurmain, R. 1991. Degenerative changes in peripheral joints as indicators of mechanical stress: Opportunities and limitations International Journal of Osteoarchaeology 1 (3-4):247-252.

Jurmain, R., F. Alves-Cardoso, C. Henderson y S. Villotte 2012. Bioarchaeology's Holy Grail: The reconstruction of activity. En A Companion to Paleopathology, editado por A. Grauer, pp. 531-552. Wiley-Blackwell, Chichester.

Jurmain, R. y L. Kilgore 1995. Skeletal evidence of osteoarthritis: A paleopathological perspective. Annals of the Rheumatic Diseases 53 (6):443-450

Klaus, H. 2016. Vida y muerte en el Perú colonial: Inicios de la bioarqueología en Lambayeque histórico (1536-1750 dC). Boletín de Arqueología PUCP 20:103-128.

Klaus, H., C. Larsen y M. Tam 2009. Economic intensification and degenerative joint disease: Life and labor on the postcontact north coast of Peru. American Journal of Physical Anthropology 139 (2):204-221.

Knüsel, C., S. Göggel y D. Lucy 1997. Comparative Degenerative Joint Disease of the Vertebral Column in the Medieval Monastic Cemetery of the Gilbertine Priory of St. Andrew, Fishergate, York, England. American Journal of Physical Anthropology 103 (4):481495 .

Lai, P. y N. Lovell 1992. Skeletal markers of occupational stress in the fur trade: A case study from a Hudson's Bay Company fur trade post. International Journal of Osteoarchaeology 2 (3):221-234.

Larsen, C. 1997. Bioarchaeology: Interpreting Behavior from the Human Skeleton. Cambridge University Press, Cambridge.

Li, G., J. Yin, J. Gao, T. Cheng, N. Pavlos, C. Zhang y M. Zheng 2013. Subchondral bone in osteoarthritis: insight into risk factors and microstructural changes. Arthritis Research \& Therapy 15 (6):223.

Litwic, A., M. Edwards, E. Dennison y C. Cooper 2013. Epidemiology and burden of osteoarthritis. British Medical Bulletin 105 (1):185-199.

Llagostera, V. 2017. Enfermedad degenerativa articular en la población elite y no elite del sitio arqueológico Solcor-3: Periodo Medio, San Pedro de Atacama, norte de Chile. Revista del Museo de Antropología 10 (2):123-130.

López, A. 1939. Clasificación de los trabajadores. El Mes Financiero y Económico, la revista para comerciantes, industriales y hombres de negocios 21:5-14

López-Barrales R., M. Hubbe, E. Aspillaga, W. Neves y H. Niemeyer 2015. Osteofitosis vertebral en poblaciones prehispánicas 
de San Pedro de Atacama, norte de Chile. Estudios Atacameños 50:177-194.

López-Bejarano, P. 2019. Gente Ociosa y Malentretenida. Trabajo y Pereza en Santafé de Bogotá, Siglo XVIII. Universidad de los Andes, Bogotá.

López-Uribe, M. 2011. Salarios, Vida Cotidiana y Condiciones de Vida en Bogotá durante la Primera Mitad del Siglo XX. Universidad de los Andes, Bogotá.

Lovell, N. 1994. Spinal arthritis and physical stress at Bronze Age Harappa. American Journal of Physical Anthropology 93 (2):149-164.

Mansegosa, D. 2016. Estudios sobre salud y enfermedad en poblaciones históricas urbanas de Mendoza (Argentina). Nuevos aportes para el estudio bioarqueológico de La Caridad. Comechingonia Virtual: Revista Electrónica de Arqueología 20 (1):111-142.

Martín, J., J. Rivera-Sandoval y C. Rojas-Sepúlveda 2009. Bioarqueología: Su aporte al proyecto arqueológico Panamá Viejo. Canto Rodado: Revista especializada en patrimonio 4:117-146.

Martínez, A. 1991. Sastres y modistas: Notas alrededor de la historia del traje en Colombia. Boletín Cultural y Bibliográfico 28 (28):61-76

Mazza, B. 2015. Estudio de los Patrones de Variación Morfológica en Restos Humanos del Humedal del Paraná Inferior: Inferencias acerca de las Pautas de Diferenciación Social en Sociedades Cazadoras-Recolectoras del Holoceno Tardío a Partir de Marcadores Óseos de Actividad. Tesis Doctoral, Facultad de Filosofía y Letras, Universidad de Buenos Aires, Buenos Aires.

Meindl, R. y O. Lovejoy 1985. Ectocranial suture closure: A revised method for the determination of skeletal age at death based on the lateral-anterior sutures. American Journal of Physical Anthropology 68 (1):57-66

Mendonça, O. y M. Arrieta 2011. Enfermedad degenerativa articular y uso del cuerpo en Rincón Chico 21 (Santa María, Catamarca)/ Degenerative joint disease and body use in Rincón Chico 21 (Santa María, Catamarca). Revista Argentina de Antropología Biológica 13 (1):03-17.

Merbs, C. 1983. Patterns of Activity-Induced Pathology in a Canadian Inuit Population. University of Ottawa Press, Ottawa.

Moreno Estefanell, L., M. D’Angelo del Campo, M. Campo Martín, P. García Laborde, Ó. Cambra-Moo, A. González Martín y R. Guichón 2018. Aproximación a la paleopatología de la columna vertebral en el cementerio de la Misión Salesiana "Nuestra Señora de la Candelaria" (S. XIX-XX, Río Grande, Argentina). Revista Argentina de Antropología Biológica 20 (2):6.

Muñoz, C. 1990. Los chinos bogotanos a comienzos de siglo (19001930): un problema vigente. Credencial Historia 12 (1 diciembre). http://www.banrepcultural.org/biblioteca-virtual/credencial-historia/ numero-12/los-chinos-bogotanos-comienzos-de-siglo-1900-1930 (8 febrero 2019).

Nathan, H. 1962. Osteophytes of the vertebral column: An anatomical study of their development according to age, race, and sex with considerations as to their etiology and significance. Journal of Bone \& Joint Surgery 44 (2):243-268.

Novak, M. y M. Šlaus 2011. Vertebral pathologies in two early modern period (16th-19th century) populations from Croatia. American Journal of Physical Anthropology 145 (2):270-281.

Ortner, D. 2003. Identification of Pathological Conditions in Human Skeletal Remains. 2da edición. Academic Press, London.

Pechenkina, E. y M. Delgado 2006. Dimensions of health and social structure in the early intermediate period cemetery at Villa
El Salvador, Peru. American Journal of Physical Anthropology 131 (2):218-235.

Ponce, P. 2010. A Comparative Study of Activity-Related Skeletal Changes in 3rd-2nd Millennium BC Coastal Fishers and 1st Millenium AD Inland Agriculturists in Chile, South America. Doctoral Dissertation, Department of Archaeology, Durham University, Durham.

Quevedo, S. 2000. Patrones de actividad a través de las patologías en población arcaica de Punta Teatinos, Norte Semiárido Chileno. Chungara Revista de Antropología Chilena 32 (1):11-21.

Rathbun, T. 1987. Health and disease at a South Carolina plantation: 1840-1870. American Journal of Physical Anthropology 74 (2):239-253.

Resnick, D. 2002. Degenerative disease of the spine. En Diagnosis of Bone and Joint Disorders, 4ta edición, editado por D. Resnick, pp. 1382-1475. WB Saunders, Philadelphia.

Rivera-Sandoval, J. 2014. Espacios mortuorios y bioarqueología histórica en la Iglesia La Candelaria en Bogotá. Maguaré 28 (2):147-174.

Rodríguez J. 1994. Perfil paleodemográfico muisca. El caso del cementerio de Soacha, Cundinamarca. Maguaré 10:7-36.

Rodríguez, J. 1999. Los Chibchas: Pobladores Antiguos de los Andes Orientales. Aspectos Bioantropológicos. ColcienciasUniversidad Nacional de Colombia, Bogotá.

Rogers, J. y T. Waldron 1995. A field Guide to Joint Disease in Archaeology. John Wiley \& Sons, Chichester.

Rogers, J., T. Waldron, P. Dieppe e I. Watt 1987. Arthropathies in palaeopathology: The basis of classification according to most probable cause. Journal of Archaeological Science 14 (2):179-193.

Rojas-Sepúlveda, C. 2009. Relations Homme-Environnement en Amérique du SudPrécolombienne: Approche Paléoépidémiologique de la Maladie Articulaire Dégénérative et des Marqueurs Osseux d'activités chez des Populations Anciennes des Régions Andines Septentrionales et Panaméennes. Thèse de Doctorat, Unité d'anthropologie Laboratoire Adaptabilité biologique et culturelle, Université d'Aix Marseille II, Marseille.

Rojas-Sepúlveda, C., Y.Ardagna y O. Dutour 2008. Paleoepidemiology of vertebral degenerative disease in a Pre-Columbian Muisca series from Colombia. American Journal of Physical Anthropology 135 (4):416-430.

Rojas-Sepúlveda, C. y O. Dutour 2014. Enfermedad articular degenerativa y cambios entesiales en seis colecciones óseas prehispánicas del noroccidente de América del Sur. Chungara Revista de Antropología Chilena 46 (1):153-169.

Rojas-Sepúlveda, C. y J. Rodríguez 2017. Informe final del proyecto: Análisis bioarqueológico de contextos funerarios del Cementerio Central, Bogotá: Vida y muerte a final del siglo XIX y principios del $X X$. Informe final presentado al ICANH. Universidad Nacional de Colombia - IDPC, Bogotá.

Rojas-Sepúlveda, C. y F. Suescún 2018. Informe de avance del proyecto: la actividad física y la alimentación en una muestra de habitantes de Bogotá del siglo XIX y XX. Informe presentado a la Dirección de Investigación y Extensión de la sede Bogotá, Convocatoria de Investigación Orlando Fals Borda - 2018 Apoyo a Proyectos de Investigación Docentes de la Facultad de Ciencias Humanas de la Universidad Nacional de Colombia sede Bogotá. Universidad Nacional de Colombia, Bogotá.

Rothschild, B. 1997. Porosity: A curiosity without diagnostic significance. American Journal of Physical Anthropology 104 (4):529-533. 
Scabuzzo, C. 2012. Estudios bioarqueológicos de marcadores de estrés ocupacional en cazadores recolectores pampeanos del Holoceno Temprano-Medio. Análisis de la serie esqueletal de Arroyo Seco 2/Occupational stress markers bioarchaeological studies of early-middle holocene. Revista Argentina de Antropología Biológica 14 (1):17-31.

Silva-Pinto, V., P. Méndez-Quiros y C. Soto 2017. Bioarqueología en la iglesia colonial de Huaviña. Quebrada de Tarapacá, Norte de Chile Boletín del Museo Nacional de Historia Natural 66 (1):29-49.

Sofaer Derevenski, J. 2000. Sex differences in activity-related osseous change in the spine and the gendered division of labor at Ensay and Wharram Percy, UK. American Journal of Physical Anthropology 111 (3):333-354.

Song, J., R. Chang y D. Dunlop 2006. Population impact of arthritis on disability in older adults. Arthritis and Rheumatism 55 (2):248-255.

Stirland, A. y T. Waldron 1997. Evidence for activity related markers in the vertebrae of the crew of the Mary Rose. Journal of Archaeological Science 24 (4):329-335.

Suby, J. y D. Giberto 2019. Temporomandibular joint osteoarthritis in human ancient skeletal remains from Late Holocene in southern Patagonia. International Journal of Osteoarchaeology 29 (1):14-25.

Suescún, F. 2017. Los Trabajadores Pobres de Bogotá, Siglos XIX y XIX: Evaluación de la Enfermedad Vertebral Degenerativa en la Colección Ósea del Cementerio Central de Bogotá. Monografía de pregrado. Departamento de Antropología, Universidad Nacional de Colombia, Bogotá.

Tiesler, V., P. Zabala y A. Cucina 2010. Natives, Europeans, and Africans in Colonial Campeche: History and Archaeology. University Press of Florida, Florida.
Tovar, H. 1995. Que nos Tengan en Cuenta: Colonos, Empresarios y Aldeas: Colombia 1800-1900. Ediciones Uniandes-Universidad de los Andes, Bogotá.

Trinkaus, E. 1985. Pathology and the posture of the La Chapelleaux-Saints Neandertal. American Journal of Physical Anthropology 67 (1):19-41.

Ubelaker, D. 1994. Biología de los Restos Humanos Hallados en el Convento de San Francisco. Instituto Nacional de Patrimonio Cultural del Ecuador, Quito.

Waldron, T. 1991. Prevalence and distribution of osteoarthritis in a population from Georgian and early Victorian London. Annals of the Rheumatic Diseases 50 (5):301-307.

Waldron, T. 1994. Counting the Dead: The Epidemiology of Skeletal Populations. John Wiley \& Sons, Chichester.

Waldron, T. y J. Rogers 1991. Inter-observer variation in coding osteoarthritis in human skeletal remains. International Journal of Osteoarchaeology 1 (1):49-56.

Watkins, R. 2012. Variation in health and socioeconomic status within the W. Montague Cobb skeletal collection: Degenerative joint disease, trauma and cause of death. International Journal of Osteoarchaeology 22 (1):22-44.

Woo, E. y S. Pak 2014. The relationship between the two types of vertebral degenerative joint disease in a Joseon Dynasty population, Korea. International Journal of Osteoarchaeology 24 (6):675-687.

Zampetti, S., V. Mariotti, N. Radi y M. Belcastro 2016. Variation of skeletal degenerative joint disease features in an identified Italian modern skeletal collection. American Journal of Physical Anthropology 160 (4):683-693. 